\title{
Kaum Einschränkungen bei Privatpatienten
}

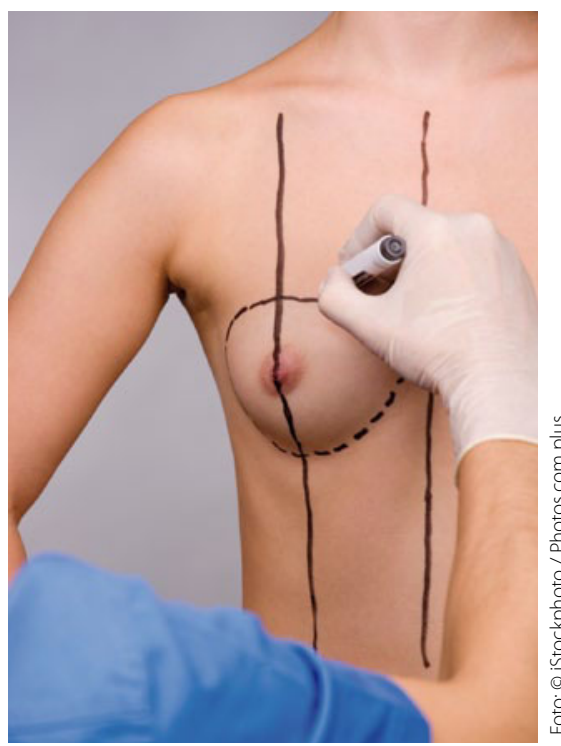

Privat abgerechnet, kann der Mund-Kiefer-Gesichtschirurg auch hier operieren.
- Das Bundesverfassungsgericht hat die ärztliche Beschränkung auf das Fachgebiet, wie sie in den Berufsordnungen und nahezu bundesweit auch in den Heilberufe- und Kammergesetzen der Länder festgeschrieben ist, verworfen (Az.: 1 BvR 2383/10). „Diese müssen so ausgelegt werden, dass Ausnahmen zulässig sind", kommentiert Rechtsanwalt Felix Heimann von der Kanzlei kwm in Hamburg.

Der klagende Facharzt für MundKiefer-Gesichtschirurgie ist Arzt und Zahnarzt. In seiner Praxis führt er 3600 Operationen im Mund-, Kiefer- und Gesichtsbereich pro Jahr durch. Daneben fungiert er als Geschäftsführer einer Klinik für Schönheitsoperationen. 400 bis 500 Eingriffe fallen dort jährlich an. Etwa ein Zehntel davon entfällt auf das
Einsetzen von Brustimplantaten sowie Bauch- und Oberarmstraffungen. Das Hamburger Heilberufe-Gericht erteilte Ende 2009 einen Verweis und setzte eine Geldbuße von 1500 Euro fest. 2010 bestätigte der Berufsgerichtshof dieses Urteil. In seiner Verfassungsbeschwerde machte der Arzt geltend, nur etwa 1,5\% seiner Eingriffe seien fachfremd. Hierfür sei er aber aufgrund seiner Doppelapprobation und Fortbildungen ausreichend qualifiziert.

Die vertragsärztliche Versorgung betrifft das Urteil kaum. Anders sieht es aber bei IGeL und Privatleistungen aus. Zwar ist auch die GOÄ nach Fachgebieten gegliedert, „daraus ergibt sich aber keine Beschränkung für die Ärzte", erläutert Dirk Lullies, Sprecher des PKVVerbandes in Berlin.

MWO

\section{Für die Bank zählen auch die Soft-Facts}

- Bevor Ärzte einen Kredit erhalten, werden nicht nur ihre Finanzen von den Banken genau unter die Lupe genommen. Beim bankinternen Rating spielen vor allem die Soft-Facts eine immer größere Rolle. Darauf hat die Initiative Finanzstandort Deutschland (IFD) hingewiesen. Dabei erwarten die Banken un- ter anderem folgende Angaben von Ärzten: eine Darstellung der Markt- und Wettbewerbssituation, Infos zur Managementqualität des Arztes (gibt es eine Liquiditätsplanung und ein funktionierendes Personalmanagement?) sowie einen Soll-Ist-Vergleich zu „Zukunftsmaßnahmen“ der Praxis.

REH -

\section{Serviceleistungen und Beratung für die Patienten immer wichtiger}

- Die Servicequalität in Praxen wird für Patienten zunehmend wichtig. Das ergibt die IFABS-Analyse von Patientenbefragungen bei niedergelassenen Ärzten aus den Jahren 2000 und 2010. 100 Befragungen in Arztpraxen aus den beiden Jahren wurden verglichen und fast 10000 Fragebögen einbezogen. Bewerteten Patienten Betreuung und Praxisleistung 2000 und 2010 fast gleich, legten sie jetzt stärker Wert auf Ambiente, Bedienung, Beratung, Professionalität, Komfort, Kompetenz, Freundlichkeit und Service.

\section{Online-Terminvergabe}

\section{Ein (zu?) seltenes Serviceangebot}

— Die Möglichkeit, Termine online zu vergeben, hat sich in Arztpraxen noch nicht durchgesetzt. Nur 14\% der Ärzte bieten diesen Service an. Dies hat die Studie „Ärzte im Zukunftsmarkt Gesundheit 2010“ der Stiftung Gesundheit ergeben. Sie ergab auch, dass immerhin mehr als die Hälfte der befragten Ärzte, Zahnärzte und Psychologischen Psychotherapeuten darin durchaus einen Service für Patienten erkennen. Ein Grund für den geringen Verbreitungsgrad von Online-Terminen ist unter anderem, dass Ärzte eine Schieflage im KostenNutzen-Verhältnis sehen. 